\title{
Inherited ichthyoses: molecular causes of the disease in Czech patients
}

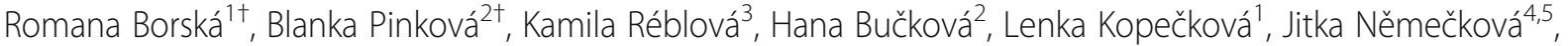 \\ Alena Puchmajerová ${ }^{6,7}$, Marcela Malíková ${ }^{6}$, Markéta Hermanová ${ }^{8}$ and Lenka Fajkusová1,3,9*
}

\begin{abstract}
Inherited ichthyoses belong to a large and heterogeneous group of mendelian disorders of cornification, and can be distinguished by the quality and distribution of scaling and hyperkeratosis, by other dermatologic and extracutaneous involvement, and by inheritance. We present the genetic analysis results of probands with X-linked ichthyosis, autosomal recessive congenital ichthyosis, keratinopathic ichthyosis, and a patient with Netherton syndrome. Genetic diagnostics was complemented by in silico missense variant analysis based on 3D protein structures and commonly used prediction programs to compare the yields of these two approaches to each other. This analysis revealed various structural defects in proteins coded by mutated genes while no defects were associated with known polymorphisms. Two patients with pathogenic variants in the ABCA12 gene have a premature termination codon mutation on one allele and a silent variant on the second. The silent variants c.69G > A and c.4977G > A are localised in the last nucleotide of exon 1 and exon 32, respectively, and probably affect mRNA splicing. The phenotype of both patients is very severe, including a picture harlequin foetus after birth; later (at 3 and 6 years of age, respectively) ectropin, eclabion, generalised large polygonal scaling and erythema.
\end{abstract}

Keywords: Autosomal recessive congenital ichthyosis, Keratinopathic ichthyosis, In silico analysis, 3D protein structure

\section{Dear Editor,}

Inherited ichthyoses are a heterogeneous group of disorders classified by the quality and distribution of scaling and hyperkeratosis, by other dermatologic and extracutaneous involvement, and by inheritance [1]. The aim of our study was identify variants in genes related to an ichthyosis phenotype and evaluate their pathogenicity. From this reason, we introduced targeted sequencing of 180 genodermatosis related genes (20 of which associated with an ichthyosis - STS, ABCA12, ALOXE3, ALOX12B, CERS3, CYP4F22, LIPN, NIPAL4, POMP, PNPLA1, TGM1, KRT1, KRT2, KRT10, ABHD5, ALDH3A2, GJB2, SLC27A4, SPINK5, ST14) and structural analysis of 3D proteins.

\footnotetext{
*Correspondence: lenka.fajkus@gmail.com

${ }^{\dagger}$ Romana Borská and Blanka Pinková contributed equally to the paper as first authors.

${ }^{1}$ Centre of Molecular Biology and Gene Therapy, University Hospital Brno and Masaryk University, Jihlavská 20, 62500 Brno, Czech Republic

${ }^{3}$ Central European Institute of Technology, Masaryk University, Kamenice 753/ 5, 62500 Brno, Czech Republic

Full list of author information is available at the end of the article
}

Overall, we found 47 probands with autosomal recessive congenital ichthyosis (ARCI), 9 probands with keratinopathic ichthyosis (KI), 2 probands with X-linked ichthyosis (XLI, patients with the STS gene deletion are not included), and one proband with Netherton syndrome (Table 1). The patients' clinical findings are presented in Additional file 1. From 47 ARCI patients, 18 patients (38.3\%) have pathogenic sequence variants in $A L O X 12 B, 9$ patients (19.1\%) in ALOXE3, 6 patients (12.8\%) in NIPAL4, 5 patients (10.6\%) in CYP4F22, 6 patients (12.8\%) in TGM1, and 3 patients (6.4\%) in ABCA12. Among analysed patients' DNA, three atypical sequence changes were identified: 1 ) a large gene deletion determined by SNP array in patient 27 [p.(Pro630Leu) on the second ALOXE3 allele]; 2) the variant c.69G $>\mathrm{A}$, p.(Pro23=), r.(spl?) in patient 45 [p.(Arg1881*) on the second $A B C A 12$ allele]; 3) the variant c.4977G $>\mathrm{A}$, p.(Glu1659=), r.(spl?) in patient 46 [c.483_484delCGinsT on the second $A B C A 12$ allele]. In silico analyses of c.69G > A (the last nucleotide of exon 1) and c.4977G > A (the last nucleotide of exon 32) using the mutation analysis software Alamut Visual v.2.10.0 revealed that these

(C) The Author(s). 2019 Open Access This article is distributed under the terms of the Creative Commons Attribution 4.0 International License (http://creativecommons.org/licenses/by/4.0/), which permits unrestricted use, distribution, and 
Table 1 Pathogenic sequence variants identified in Czech probands with ichthyosis

\begin{tabular}{|c|c|c|c|}
\hline No. & Gene & 1st allele (cDNA level, protein level) & 2nd allele (cDNA level, protein level) \\
\hline 1 & $A L O X 12 B$ & c.467_470dupATGT, p.(His158Cysfs*20) & c.1562A > G, p.(Tyr521Cys) \\
\hline 2 & $A L O X 12 B$ & c.581A > T, p. (Lys194*) & c.1562A > G, p.(Tyr521Cys) \\
\hline 3 & $A L O X 12 B$ & c.665A > T, p.(Lys222lle) & c.1562A > G, p.(Tyr521Cys) \\
\hline 4 & $A L O X 12 B$ & c.787_789delTTC, p.(Phe262del) & c.1562A > G, p.(Tyr521Cys) \\
\hline 5 & $A L O X 12 B$ & c.1034-1035delTT, p.(Phe345Trpfs*28) & c.1790C > A, p.(Ala597Glu) \\
\hline 6 & $A L O X 12 B$ & $1071 \mathrm{G}>\mathrm{C}, \mathrm{p} .(\mathrm{G} \ln 357 \mathrm{His})$ & c. $1654+3 A$ > G, r.(spl?) \\
\hline 7 & $A L O X 12 B$ & c. $1156 C$ > T, p.(Arg386Cys) & c. $1654+3 A$ > G, r.(spl?) \\
\hline 8 & $A L O X 12 B$ & c.1156C > T, p.(Arg386Cys) & c.1790C > A, p.(Ala597Glu) \\
\hline 9 & $A L O \times 12 B$ & c.1157G > A, p.(Arg386His) & c.1265C > T, p.(Pro422Leu) \\
\hline 10 & $A L O X 12 B$ & c. $1157 G$ > A, p.(Arg386His) & c.1562A > G, p.(Tyr521Cys) \\
\hline 11 & $A L O X 12 B$ & c.1294C > T, p.(Arg432*) & c.1562A > G, p.(Tyr521Cys) \\
\hline 12 & $A L O X 12 B$ & c.1405C > T, p.(Arg469Trp) & c.1454_1455delTT, p.(Phe485Cysfs*16) \\
\hline 13 & $A L O X 12 B$ & c.1448A > G, p.(Asn483Ser) & c.1562A > G, p.(Tyr521Cys) \\
\hline 14 & $A L O X 12 B$ & c.1496G > A, p.(Arg499His) & c.1496G > A, p.(Arg499His) \\
\hline 15 & $A L O X 12 B$ & c.1562A > G, p.(Tyr521Cys) & c.1688 T > C, p.(Leu563Pro) \\
\hline 16 & $A L O X 12 B$ & c.1562A > G, p.(Tyr521Cys) & c.1790C > A, p.(Ala597Glu) \\
\hline 17 & $A L O X 12 B$ & c.1562A > G, p.(Tyr521Cys) & c.1790C > A, p.(Ala597Glu) \\
\hline 18 & $A L O X 12 B$ & c.1918delG, p.(Asp640Thrfs*23) & c.1918delG, p.(Asp640Thrfs*23) \\
\hline 19 & ALOXE3 & c.36_39delACCT, p.(Tyr13*) & c.700C > T, p. $\left(\operatorname{Arg} 234^{*}\right)$ \\
\hline 20 & ALOXE3 & c.700C > T, p. $\left(\operatorname{Arg} 234^{*}\right)$ & c.700C > T, p. $\left(\operatorname{Arg} 234^{*}\right)$ \\
\hline 21 & ALOXE3 & c.700C > T, p.(Arg234*) & c.700C > T, p.(Arg234*) \\
\hline 22 & ALOXE3 & c.700C > T, p. $\left(\operatorname{Arg} 234^{*}\right)$ & c.700C > T, p. $\left(\operatorname{Arg} 234^{*}\right)$ \\
\hline 23 & ALOXE3 & c.700C > T, p. $\left(\operatorname{Arg} 234^{*}\right)$ & c.1889C > T, p.(Pro630Leu) \\
\hline 24 & ALOXE3 & c.700C > T, p. $\left(\operatorname{Arg} 234^{*}\right)$ & c.1889C > T, p.(Pro630Leu) \\
\hline 25 & ALOXE3 & c. 1392 + 2 T > A, r.spl? & c.1889C > T, p.(Pro630Leu) \\
\hline 26 & ALOXE3 & c.1889C > T, p.(Pro630Leu) & c.2097C > T, p.(Tyr699*) \\
\hline 27 & ALOXE3 & c.1889C > T, p.(Pro630Leu) & gross deletion \\
\hline 28 & NIPAL4 & c.527C > A, p.(Ala176Asp) & c.527C > A, p.(Ala176Asp) \\
\hline 29 & NIPAL4 & c.527C > A, p.(Ala176Asp) & c.527C > A, p.(Ala176Asp) \\
\hline 30 & NIPAL4 & c.527C > A, p.(Ala176Asp) & $\begin{array}{l}\text { c.1010_1015dupTCAGCA, } \\
\text { p.(Ser338_Thr339inslleSer) }\end{array}$ \\
\hline 31 & NIPAL4 & c.527C > A, p.(Ala176Asp) & c.1193dupT, p.(Val401Argfs*36) \\
\hline 32 & NIPAL4 & c.1063delC, p.(Leu355Trpfs*93) & c.1063delC, p.(Leu355Trpfs*93) \\
\hline 33 & NIPAL4 & c.1112C > G, p.(Ser371Leu) & c.1112C > G, p.(Ser371Leu) \\
\hline 34 & CYP4F22 & c.1A > G, (p. Met1?) & c.59dupG, p.(Ile21Hisfs*59) \\
\hline 35 & CYP4F22 & c.59dupG, p.(Ile21Hisfs*59) & c.59dupG, p.(Ile21Hisfs*59) \\
\hline 36 & CYP4F22 & c.59dupG, p.(Ile21Hisfs*59) & c.59dupG, p.(Ile21Hisfs*59) \\
\hline 37 & CYP4F22 & c.844C > T, p.(Arg282Trp) & c.1085G > A, p.(Arg362GIn) \\
\hline 38 & CYP4F22 & c. $1085 G$ > A, p.(Arg362Gln) & c.1085G > A, p.(Arg362Gln) \\
\hline 39 & TGM1 & c.376C > T, p.(Arg126Cys) & c.919C > T, p.(Arg307Trp) \\
\hline 40 & TGM1 & c.377G > A, p.(Arg126His) & c.377G > A, p.(Arg126His) \\
\hline 41 & TGM1 & c.425G > A, p.(Arg142His) & c.1184C > T p.(Thr395Ile) \\
\hline 42 & TGM1 & c.425G > A, p.(Arg142His) & c.2000 T > G, p.(Leu667Arg) \\
\hline 43 & TGM1 & c.968G > A, p.(Arg323Gln) & c.1135G > C, p.(Val379Leu) \\
\hline
\end{tabular}


Table 1 Pathogenic sequence variants identified in Czech probands with ichthyosis (Continued)

\begin{tabular}{|c|c|c|c|}
\hline No. & Gene & 1st allele (cDNA level, protein level) & 2nd allele (cDNA level, protein level) \\
\hline 44 & TGM1 & c.1310 T > G, p.(Val437Gly) & c.2307C > G, p.(Ser769Arg) \\
\hline 45 & $A B C A 12$ & c.69G > A, p.(Pro23=), r.(spl?) & c.5641C > T, p. $\left(\operatorname{Arg} 1881^{*}\right)$ \\
\hline 46 & $A B C A 12$ & c.483_484delCGinsT, p.(Ala162Hisfs*10) & c.4977G > A, p.(Glu1659=), r.(spl?) \\
\hline 47 & $A B C A 12$ & c.2634C > G, p.(Phe878Leu) & c.4139A > G, p.(Asn1380Ser) \\
\hline 48 & KRT1 & c.532 T> C, p.(Ser178Pro) ${ }^{a}$ & - \\
\hline 49 & KRT1 & c.593C > T, p. $(\text { Val198Gly })^{\text {b }}$ & - \\
\hline 50 & KRT1 & c.1016delT, p.(Met339Argfs*23) & - \\
\hline 51 & KRT10 & c. $467 \mathrm{G}>\mathrm{A}, \mathrm{p} .(\operatorname{Arg} 156 \mathrm{His})^{\mathbf{b}}$ & - \\
\hline 52 & KRT10 & c. $467 \mathrm{G}>\mathrm{A}$, p. $(\operatorname{Arg} 156 \mathrm{His})^{\mathbf{b}}$ & - \\
\hline 53 & KRT10 & c. $1373+1 G>C$, r.spl? & - \\
\hline 54 & KRT10 & c.1374-1G > C, r.spl? & - \\
\hline 55 & KRT2 & c.1435A > C, p.(Thr479Pro) ${ }^{c}$ & - \\
\hline 56 & KRT2 & c.1459G > A, p.(Glu487Lys) ${ }^{c}$ & - \\
\hline 57 & STS & c. $1330 \mathrm{C}>\mathrm{T}$, p.(His444Tyr) & - \\
\hline 58 & STS & c. $1338 \mathrm{C}>$ G, p.(Cys446Trp) & - \\
\hline 59 & SPINK5 & c. $81+1 \mathrm{G}>\mathrm{A}$, r.spl? & c.1431-12G > A, r.(spl?) \\
\hline
\end{tabular}

Variants in bold letters were detected only in Czech patients (31 patients were mentioned in our previous study [2]). Genes, reference sequences: $A L O X 12 B$, NM_001139.2; ALOXE3, NM_021628.2; CYP4F22, NM_173483.3; NIPAL4, NM_001099287.1; TGM1, NM_000359.2; ABCA12, NM_173076.2; KRT1, NM_006121.3; KRT10, NM_000421.3; KRT2, NM_000423.2; STS, NM_000351.5; SPINK5, NM_006846.3. The localisation of variants in a keratin molecule: athe head domain, subdomain H1; ${ }^{b}$ the central rod domain, subdomain $1 \mathrm{~A}$, helix initiating motive; ${ }^{c}$ the central rod domain, subdomain $2 \mathrm{~A}$, helix terminating motif (www.interfil.org)
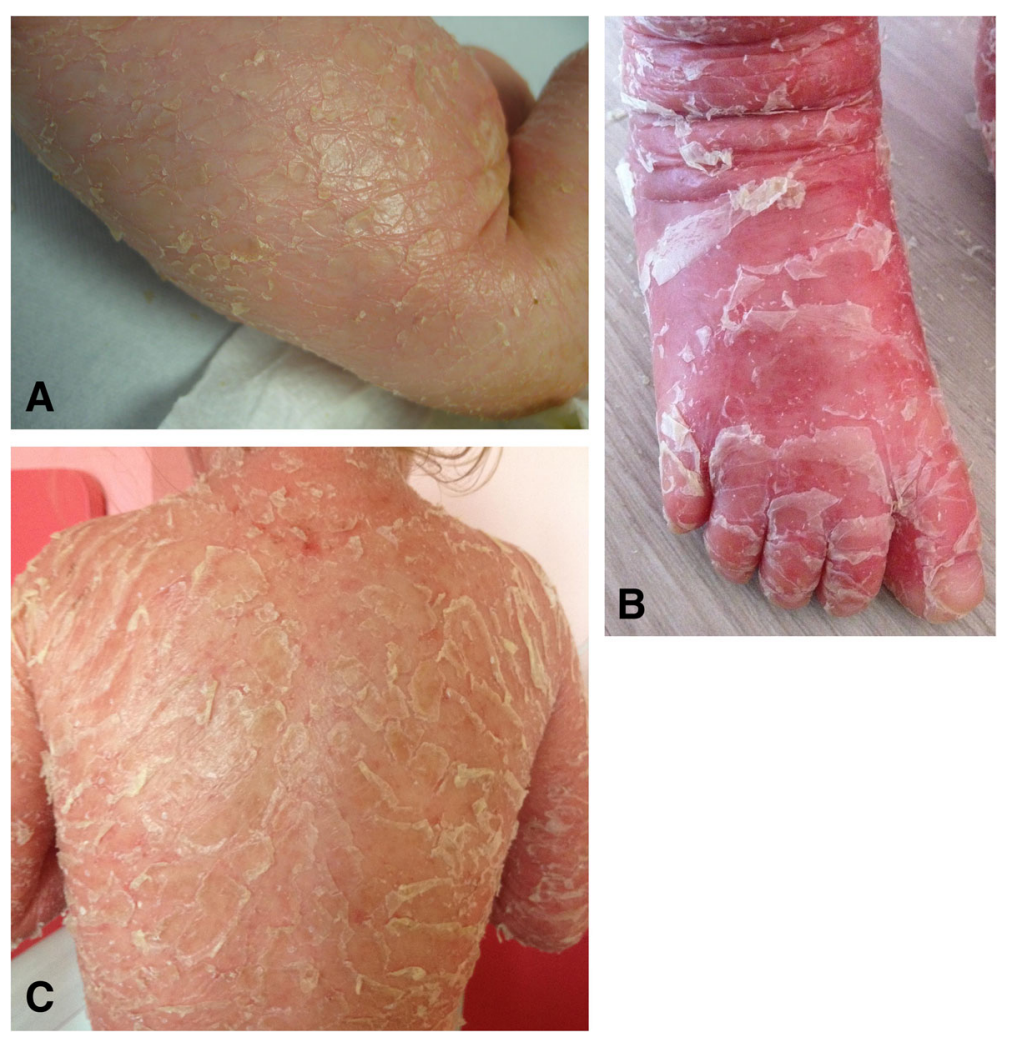

Fig. 1 Photos of patient 45 at the age of 3 months (a) and 3 years $(\mathbf{b}, \mathbf{c})$ 
variants probably affect splicing on the basis of a weakening of the authentic $5^{\prime}$ donor splice sites of exon 1 ($25.4 \%$ ) and exon $32(-69.4 \%)$, respectively.

$A B C A 12$ disease-causing variants have been described in ARCI including harlequin ichthyosis (HI), congenital ichthyosiform erythroderma (CIE), and lamellar ichthyosis (LI) [3]. HI shows the most severe phenotype and most mutations associated with this phenotype create a premature termination codon (PTC). CIE and LI are clinically characterized by fine, whitish scales on a background of erythematous skin, and large, thick, dark scales over the entire body without a serious background erythroderma, respectively. We have three patients with pathogenic variants in $A B C A 12$, two of them have a PTC mutation and a silent variant as a second mutation, but probably affecting mRNA splicing. The phenotype of both patients is very severe, including a picture harlequin foetus after birth; later (at 3 and 6 years of age, respectively) ectropin, eclabion, generalised large polygonal scaling and erythema (Fig. 1). To explore the possible association of the disease with the $A B C A 12$ gene, immunohistochemical $\mathrm{ABCA} 12$ protein analysis was performed in the case of patient 45. This analysis revealed deficient ABCA12 expression in the patient compared with normal skin tissue (Fig. 2). Patient 47, with two $A B C A 12$ missense mutations, has a milder phenotype with clinical findings corresponding to classical CIE.

From 9 KI patients, 3 patients have a pathogenic sequence variant in KRT1, 4 patients in KRT10, and 2 patients in KRT2. Mutations in the mentioned KRT genes have autosomal dominant inheritance. Unfortunately, the parent's DNA was not available in patients 48, 51, and 52 (but in all cases the identified variants were already described in HGMD as disease-causing). In patients 49,53 , and 56 , a pathogenic variant was present in one of the parents in agreement with clinical symptoms. In patient 50, 54, and 55 pathogenic variants arose as a de novo event.

As multiple genes are linked to an ichthyosis phenotype, massive parallel sequencing is effective technique for molecular genetic diagnostics. This methodical approach generates a large amount of data which need to be interpreted from the point of view of their potential disease association. We performed in silico analysis of sequence variants based on 3D protein structures and commonly used prediction programs (such as SIFT, PolyPhen-2, and MutationTaster) to evaluate the effect of 76 pathogenic and 9 benign missense variants and compare the yields of these two approaches to each other. Suitable 3D protein structures from the Protein Data Bank (https://www.rcsb.org/) were found for the STS, ALOX12B, ALOXE3, and TGM1 proteins. Pathogenic sequence variants were either identified in our patients or reported in the Human Gene Mutation
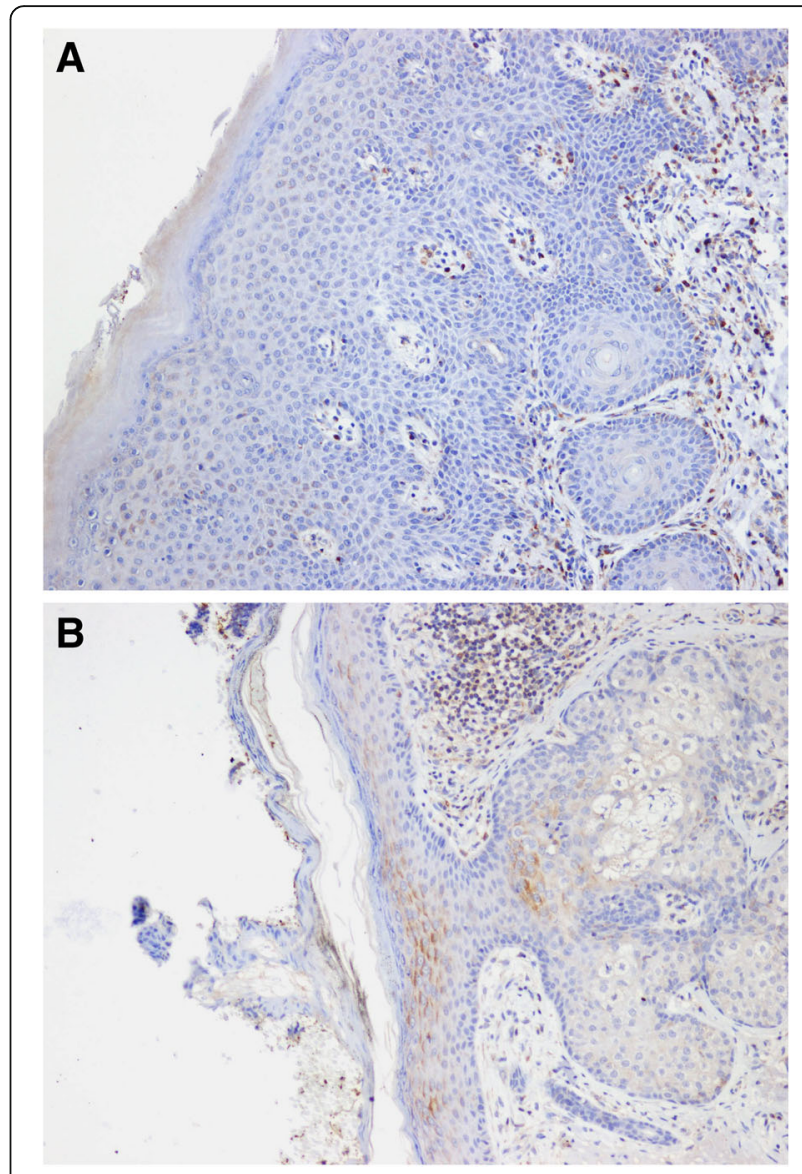

Fig. 2 Immunohistochemical detection of the ABCA12 protein in skin tissue of the patient 45 (a) and a control (b), original magnification $\times 100$

Database. Benign sequence variants were described in literature and/or indicated in the ExAC database (http:// exac.broadinstitute.org) with an allele frequency $>1 \%$. This strategy has been used in our previous studies [4-6].

Based on 3D protein structures, we were able to explain a deleterious effect of 74 pathogenic variants (97.4\%). The structural defect of sequence variants was caused by a loss of structural contacts (i.e. direct $\mathrm{H}$-bonds, salt bridges, stacking interactions), a change in physico-chemical properties, or their combinations. Considering benign variants, we observed that they are associated with no structural defects, and they are mostly localised on the protein's surface. The structural analysis is described in more detail in Additional file 2. When we used SIFT, PolyPhen-2, and MutationTaster, the degree of compliance in prediction programs and phenotype status was $85.7 \%$ for pathogenic variants and $55.5 \%$ for benign variants, assuming that the results of all three prediction programs agree. Based on published recommendations [7], the combination of predictions from different prediction programs are considered as a single piece of evidence in sequence interpretation. If all 
of the prediction programs tested agree on the prediction, then this evidence can be counted as supporting. However, if predictions disagree, then this evidence should not be used in classifying a variant. Our results show that the detailed structural analysis of proteins is a better approach to interpret sequence variants (if an appropriate 3D protein structure is available) - the degree prediction compliance and phenotype status was $97.4 \%$ for pathogenic variants and $100 \%$ for benign variants (in contrast to $85.7 \%$ and $55.5 \%$ in commonly used prediction programs).

\section{Additional files}

Additional file 1: Table S1. Patients' clinical findings. (XLSX $23 \mathrm{~kb})$

Additional file 2: In silico analyses. Methods and results related to in silico analyses. (DOCX $71 \mathrm{~kb})$

\section{Abbreviations}

ARCl: Autosomal recessive congenital ichthyosis; CIE: Congenital ichthyosiform erythroderma; HI: Harlequin ichthyosis; KI: Keratinopathic ichthyosis; LI: Lamellar ichthyosis; PTC: Premature termination codon; XLI: X-linked ichthyosis

\section{Acknowledgments}

We would like to thank the physicians from Departments of Medical Genetics in the Czech Republic (Drs M. Vlčková, M. Simandlová, E. Vyhnálková, P. Tesner, E. Kantorová, A. Drahokoupilová, P. Solařová, J. Hyjánek, Š. Prášilová, M. Lacinová, J. Šoukalová, Z. Kalina, G. Křečková, A. Klímová) for providing us with their patients' blood samples and clinical reports.

\section{Funding}

This study was supported by the project of the Technology Agency of the Czech Republic (TE02000058), the project of the Ministry of Health of the Czech Republic (FNB RVO 65269705), the project CEITEC 2020 (LQ1601), and the project of the Grant Agency of the Czech Republic (GA16-11619S/2016).

Availability of data and materials

Not applicable

\section{Authors' contributions}

$\mathrm{BR}$ and $\mathrm{KL}$ - molecular genetic diagnostics. RK - 3D protein modelling. $\mathrm{PB}$, $\mathrm{BH}, \mathrm{NM}, \mathrm{PA}, \mathrm{MM}$ - clinical diagnostics. HM - pathological analysis. FL coordination of study, writing the manuscript. All authors read and approved the final manuscript.

\section{Ethics approval and consent to participate}

Informed consent was given to participate in this study, which was approved by the Ethics Committee of the University Hospital Brno.

\section{Consent for publication}

Written consent for publication was provided by the patients or their parents, whom we would like thank for their help.

\section{Competing interests}

The authors declare that they have no competing interests.

\section{Publisher's Note}

Springer Nature remains neutral with regard to jurisdictional claims in published maps and institutional affiliations.

\section{Author details}

'Centre of Molecular Biology and Gene Therapy, University Hospital Brno and Masaryk University, Jihlavská 20, 62500 Brno, Czech Republic. ²Department of Pediatric Dermatology, Pediatric Clinic, University Hospital Brno and Masaryk University, Jihlavská 20, 62500 Brno, Czech Republic. ${ }^{3}$ Central
European Institute of Technology, Masaryk University, Kamenice 753/5, 625 00 Brno, Czech Republic. ${ }^{4}$ Department of Medical Genetics, University Hospital Brno, Jihlavská 20, 62500 Brno, Czech Republic. ${ }^{5}$ Department of Public Health, Faculty of Medicine, Masaryk University, Kamenice 5, 62500 Brno, Czech Republic. ' Institute of Biology and Medical Genetics, University Hospital Motol, V Úvalu 84, 15006 Prague, Czech Republic. 'GENNET, Kostelní 9/292, 17000 Prague, Czech Republic. ${ }^{8}$ First Department of Pathological Anatomy, Faculty of Medicine, Masaryk University and St. Anne's University Hospital, Pekařská 664/53, 65691 Brno, Czech Republic.

'Laboratory of Functional Genomics and Proteomics, NCBR, Faculty of Science, Kotlářská 267/2, 61137 Brno, Czech Republic.

Received: 24 April 2018 Accepted: 18 April 2019

Published online: 02 May 2019

References

1. Oji V, Tadini G, Akiyama M, Blanchet Bardon C, Bodemer C, Bourrat E, et al. Revised nomenclature and classification of inherited ichthyoses: results of the first ichthyosis consensus conference in Soreze 2009. J Am Acad Dermatol. 2010;63:607-41.

2. Buckova H, Noskova H, Borska R, Reblova K, Pinkova B, Zapletalova E, et al. Autosomal recessive congenital ichthyoses in the Czech Republic. Br J Dermatol. 2016;174:405-7.

3. Akiyama M. ABCA12 mutations and autosomal recessive congenital ichthyosis: a review of genotype/phenotype correlations and of pathogenetic concepts. Hum Mutat. 2010;31:1090-6.

4. Reblova K, Hruba Z, Prochazkova D, Pazdirkova R, Pouchla S, Zeman J, et al. Hyperphenylalaninemia in the Czech Republic: genotype-phenotype correlations and in silico analysis of novel missense mutations. Clin Chim Acta. 2013:419:1-10.

5. Skalova D, Zidkova J, Vohanka S, Mazanec R, Musova Z, Vondracek P, et al. CLCN1 mutations in Czech patients with myotonia congenita, in silico analysis of novel and known mutations in the human dimeric skeletal muscle chloride channel. PLoS One. 2013;8:e82549.

6. Reblova K, Kulhanek P, Fajkusova L. Computational study of missense mutations in phenylalanine hydroxylase. J Mol Model. 2015;21:70.

7. Richards S, Aziz N, Bale S, Bick D, Das S, Gastier-Foster J, et al. Standards and guidelines for the interpretation of sequence variants: a joint consensus recommendation of the American College of Medical Genetics and Genomics and the Association for Molecular Pathology. Genet Med. 2015:17:405-24

Ready to submit your research? Choose BMC and benefit from:

- fast, convenient online submission

- thorough peer review by experienced researchers in your field

- rapid publication on acceptance

- support for research data, including large and complex data types

- gold Open Access which fosters wider collaboration and increased citations

- maximum visibility for your research: over $100 \mathrm{M}$ website views per year

At BMC, research is always in progress.

Learn more biomedcentral.com/submissions 